\title{
Prognostic Value of Electroneurography and Electromyography in Facial Palsy
}

\author{
Maria Grosheva, MD; Claus Wittekindt, MD; Orlando Guntinas-Lichius, MD
}

Objectives: To compare the prognostic value of electroneurography (ENG) and needle electromyography (EMG) to estimate facial function outcome after acute facial palsy.

Study Design: Retrospective study using electrodiagnostic data and medical chart review.

Methods: Two hundred one patients treated 1995 to 2004 were included. Initial and final facial function was established clinically by the House-Brackmann (HB) scale. ENG results were classified into amplitude loss less than $75 \%$ and amplitude loss $75 \%$ or greater to predict complete recovery and defective healing, respectively. Initial and follow-up EMG results were classified into neurapraxia and predicted complete recovery. In contrast, axonotmesis/neurotmesis and mixed lesions predicted, by definition, defective healing.

Results: Initial HB was II to IV in 154 patients and $\mathrm{V}$ to VI in 47 patients. The etiology was idiopathic palsy in 139 , iatrogenic lesion in 29 , traumatic in 18 , and herpes zoster in 15 patients. Finally, $134(67 \%)$ patients showed a full recovery. Sixty-seven (33\%) patients showed signs of defective healing. ENG presented a sensitivity, specificity, accuracy, positive predictive value (to predict defective healing), and negative predictive value of $60 \%, 79 \%$, $73 \%, 59 \%$, and $80 \%$, respectively. The values for the initial EMG were $66 \%, 98 \%, 89 \%, 91 \%$, and $89 \%$. The best results showed the follow-up EMG with $85 \%, 100 \%, 97 \%$, $100 \%$, and $96 \%$. EMG results were not classifiable in 32 (16\%) patients.

Conclusions: EMG showed higher prognostic values than ENG, especially when repeated during the time course of the facial palsy. ENG might be helpful if the EMG result is not classifiable.

Key Words: Facial nerve, paralysis, electrodiagnostics, Bell's palsy, outcome.

Laryngoscope, 118:394-397, 2008

From the Department of Otorhinolaryngology, Head and Neck Surgery (M.G.), University of Cologne, Cologne, Germany; and the Department of Otorhinolaryngology (c.W.; O.G.-L.), Friedrich-Schiller-University, Jena, Germany. 9, 2007

Editor's Note: This Manuscript was accepted for publication October

Send correspondence to Dr. Orlando Guntinas-Lichius, Department of Otorhinolaryngology, Friedrich-Schiller-University Jena, Lessingstrasse 2, D-07740 Jena, Germany. E-mail: orlando.guntinas@med. uni-jena.de

DOI: $10.1097 / M L G .0 b 013 e 31815 d 8 e 68$

\section{INTRODUCTION}

Most patients with acute peripheral facial palsy are concerned about the possibility of developing a permanent facial deformity. Therefore, patients want to know its probability and the duration to recovery. ${ }^{1}$ However, evaluation of the prognosis of patients with acute facial palsy is helpful not only for counseling patients. Especially in cases of iatrogenic or traumatic lesions, evaluation of prognosis might guide further management, for instance, to answer the question of whether the nerve should be decompressed and the lesion site be exposed to repair the defect. Since the early 1970 s, when evoked electromyography (EMG) was introduced, referred to as electroneuronography or briefly as electroneurography (ENG) by Esslen, ${ }^{2}$ prognostication has mainly been based on ENG and other commonly used electrical tests such as the nerve excitability test and voluntary EMG. The value of the different electrophysiologic tests for outcome prediction of acute peripheral facial nerve palsy is still controversial. In particular, the prognostic significance of ENG and Esslen's theory that the amplitude of the compound action potential (CAP) of the facial muscles is directly proportional to the number of neurapraxic nerve fibers not undergoing degeneration is doubtful. ${ }^{1,3,4}$ On the other hand, EMG recently has been shown to be a reliable tool to predict unfavorable outcome in acute facial palsy. ${ }^{1,5}$

The purpose of our study was to compare the prognostic value of ENG and EMG in the same large, unselected series of patients with acute peripheral facial palsy with known clinical outcome who underwent a standardized diagnostic follow-up at a university-based center.

\section{PATIENTS AND METHODS}

A retrospective study was conducted. We reviewed the case records of all patients who presented with acute peripheral facial palsy at a single center (Department of Otolaryngology, Head and Neck Surgery, University of Cologne, Germany) from 1995 to 2004. This time frame was selected to provide adequate regeneration time and follow-up. Only patients with one or more facial nerve ENG examinations, two or more facial nerve EMG examinations, and 1 or more EMG examinations 14 days or later after onset of the lesions and who completed facial regeneration were included. Complete regeneration was defined as completed recovery or completed defective healing without changes during further follow-up examinations. Patients with central facial palsy, congenital palsy, and patients with surgical reconstruction of the 
facial nerve were excluded. Demographic and follow-up clinical information was obtained from the review of the patients' medical histories and the electrodiagnostic database of the department. Two hundred one patients fulfilled the inclusion criteria.

\section{Clinical Evaluation of Facial Nerve Function}

The initial and final facial nerve function was reported using the House-Brackmann (HB) facial nerve grading system. ${ }^{6}$ The HB grading system judges the facial function in six categories (grade $\mathrm{I}=$ normal function in all facial areas, to grade VI = complete paralysis plus gross asymmetry at rest).

\section{ENG and EMG Evaluation of Facial Nerve Function}

The first electrodiagnostic test including ENG and EMG was performed within 24 hours after presentation to the clinic. For stimulation and recording, a Neuroscreen plus system (Jaeger-Toennies, Inc., Hoechberg, Germany) was used. ENG was performed first on the healthy side and then repeated on the affected side. A bipolar stimulator was placed over the truncal region of the facial nerve on the skin over the stylomastoid foramen and manually adjusted to determine the best position to generate the CAP. The recording surface electrodes were placed in the nasolabial fold. Skin of the fold was prepared with abrasive conductive paste to reduce skin impedance. The ground electrode was placed around the patient's wrist. A rectangular pulse 0.2 milliseconds long with a frequency of $1 \mathrm{~Hz}$ was produced. The stimulation current intensity was increased stepwise until there was no further increase in the amplitude of the diphasic myogenic CAP. An additional $10 \%$ of current was added to ensure supramaximal stimulation. The stimulation intensity ranged from 9 to $21 \mathrm{~mA}$. The peak-to-peak amplitude was measured with the software included with the Neuroscreen plus system. The percentage of amplitude loss was calculated as $100 \times(1-$ [amplitude on affected side/amplitude on healthy side]). An amplitude loss of less than $75 \%$ was defined as a prognostic sign for complete recovery. In contrast, an amplitude loss of $75 \%$ or greater was defined as a prognostic sign for defective healing.

For EMG, the patients were examined by bipolar needle EMG of the mimic musculature of the affected side. ${ }^{1,7}$ If the first EMG examination was performed less than 14 days after onset of the palsy, a second examination later than 14 days after onset was obligatory. The patients were re-examined on a regular basis in the outpatient department. The EMG examinations were stopped when a complete recovery or a definitive defective healing was recorded. The face was examined at rest and during voluntary activity, and the function of six facial muscles was analyzed: the frontalis, orbicularis oculi, major zygomatic, orbicularis oris, levator labii superior, and the depressor anguli oris muscle. The EMG recordings were analyzed for spontaneous fibrillation potentials, the degree of voluntary polyphasic reinnervation potentials, and for synkinetic activity. The recording techniques were previously published in detail. ${ }^{1}$ The EMG recordings were classified according to Seddon. " "Neurapraxia" was defined as absence of pathologic, spontaneous fibrillation activity and decreased or lost voluntary activity. "Axonotmesis/neurotmesis" was defined as pathologic, spontaneous activity in one or more facial muscles and decreased or lost voluntary activity. Because spontaneous activity cannot be expected earlier than 10 to 14 days after onset of paralysis, the later EMG examinations were important. Because neurotmesis cannot be clearly and reliably differentiated from axonotmesis electrophysiologically, no attempt was made to distinguish between them. ${ }^{1}$ If the recording of some muscles clearly argued for neurapraxia but of other muscles for axonotmesis/neurotmesis, the recordings were classified as "mixed lesion." By definition, a neurapraxia was classified as a prognostic sign for complete recovery. In contrast, axonotmesis/ neurotmesis and mixed lesion were classified as prognostic signs for defective healing. ${ }^{5}$

\section{Treatment of Patients}

Patients with idiopathic facial palsy were treated by infusion therapy with high-dose prednisolone and pentoxifylline. ${ }^{9}$ This regime was combined with acyclovir in patients with Ramsay-Hunt syndrome. In cases of traumatic or iatrogenic origin, the therapy was determined by onset, further symptoms, and electrodiagnostic results. Surgical therapy was reserved for individual patients with early onset, ENG amplitude loss of $100 \%$, and no voluntary EMG activity. As mentioned above, patients with facial nerve reconstructions were excluded from the study.

\section{Statistical Analysis}

Statistical analysis of the data was performed using SPSS software (Chicago, IL) for medical statistics. If not otherwise given, data are presented as means $\pm \mathrm{SD}$. To compare the ENG amplitude loss in patients with complete recovery and patients with defective healing, the Mann-Whitney test was used. To evaluate the prognostic value of ENG and EMG recording, sensitivity, specificity, accuracy, positive predictive value (PPV), and negative predictive value (NPV) were calculated by means of standard formulas. For calculation of PPV and NPV, "defective healing" was defined as a positive test result. "Complete recovery" was defined as a negative test result. The McNemar test was used to compare the performances of ENG and EMG to prognose the correct outcome. To describe the concordance between ENG and EMG, the kappa statistic was used. To examine the role of the etiology on the prognostic value of ENG and EMG, Pearson's $\chi^{2}$ test was used. Nominal two-sided $P$ values are reported. The significance level was set at $P<.05$.

\section{RESULTS}

\section{Patient Characteristics and Clinical Facial Function}

The study sample consisted of 69 female patients and 132 male patients with a mean age of 51 (range, 18-90) years. The right and left side were involved in 106 and 95 patients, respectively. The etiology was idiopathic in 139 patients, iatrogenic in 29 patients, traumatic in 18 patients, and herpes zoster infection in 15 patients. The ENG and first EMG were performed $13 \pm 18$ days after onset of the palsy.

\section{Clinical Facial Nerve Function and Recovery}

The clinical facial nerve function at first presentation according to the HB scale was HB grade II in 37 patients, grade III in 27 patients, grade IV in 90 patients, grade V in 15 patients, grade VI in 29 patients, and unknown in 3 patients. One hundred thirty-four $(67 \%)$ patients showed a full recovery of the palsy. Sixty-seven (33\%) patients developed defective healing. In accordance, the final outcome was HB grade I in 134 patients, grade II in 39 patients, HB III in 13 patients, HB IV in 8 patients, HB V in 5 patients, and $\mathrm{HB}$ VI in 2 patients.

\section{ENG and EMG Results}

After supramaximal stimulation, the CAP of the ENG in the nasolabial fold was $2.1 \pm 1.6 \mathrm{mV}$ and $0.9 \pm 1.0$ $\mathrm{mV}$ on the healthy side and on the paralyzed side, respectively. In looking only at the affected side, the CAP was 
TABLE I.

Results of Electroneurography (ENG), Initial Electromyography (EMG), and Follow-Up EMG in 201 Patients.

Clinical Outcome

\begin{tabular}{lcc}
\hline & \multicolumn{2}{c}{ Clinical Outcome } \\
\cline { 2 - 3 } Method/Result & Recovery & Defective Healing \\
\hline ENG predicted recovery & 106 & 27 \\
ENG predicted defective healing & 28 & 40 \\
Initial EMG predicted recovery & 122 & 15 \\
Initial EMG predicted defective & 3 & 29 \\
$\quad$ healing & 9 & 23 \\
Initial EMG not classifiable & 128 & 35 \\
Follow-up EMG predicted recovery & 0 & 26 \\
Follow-up EMG predicted defective & & \\
$\quad$ healing & 6 & \\
Follow-up EMG not classifiable &
\end{tabular}

$1.1 \pm 1.0 \mathrm{mV}$ in patients who later showed a complete recovery in contrast to $0.7 \pm 1.0 \mathrm{mV}$ in patients who later developed defective healing. This resulted in a mean amplitude loss of $47 \%$ in patients with later recovery in contrast to a significantly larger mean amplitude loss of $71 \%$ in patients with later defective healing (MannWhitney test, $P<.0001)$. The amplitude loss was less than $75 \%$ in 133 patients and $75 \%$ or greater in 68 patients (i.e., ENG predicted a full recovery in 133 patients and defective healing in 68 patients). Compared with the final outcome, the ENG prognosis was correct in $146(73 \%)$ patients. All prognostic values are shown in Table I.

The initial EMG revealed neurapraxia in 137 patients, axonotmesis in 24 patients, and mixed lesions in 8 patients. The EMG recordings were not classifiable in 32 patients. With these results, the initial EMG predicted a full recovery in 137 patients and defective healing in 32 patients. Compared with the final outcome, the initial EMG prognosis was correct in 151 (89\%) patients. The follow-up EMG revealed a neurapraxia in 134 patients, axonotmesis in 30 patients, and mixed lesions in 5 patients. The EMG was still not classifiable in 32 patients. Compared with the final outcome, the follow-up EMG prognosis was correct in $163(97 \%)$ patients.

Sensitivity, specificity, accuracy, PPV, and NPV of the ENG and EMG results are presented in Table II. The follow-up EMG had the highest prognostic values. ENG showed poorer values. The follow-up EMG showed the

TABLE II.

Comparison of Prognostic Values of Electroneurography (ENG), Initial Electromyography (EMG), and Follow-Up EMG for Defective Healing.

\begin{tabular}{lccc}
\hline & ENG (\%) & Initial EMG (\%) & Follow-Up EMG (\%) \\
\hline Sensitivity & 60 & 66 & 85 \\
Specificity & 79 & 98 & 100 \\
Accuracy & 73 & 89 & 97 \\
PPV & 59 & 91 & 100 \\
NPV & 80 & 89 & 96 \\
\hline \hline
\end{tabular}

PPV $=$ positive predictive value; NPV $=$ negative predictive value. best performance (>initial EMG > ENG; McNemar test, $P<.001$, respectively). The concordance between the results of the different electrophysiologic tests was low (ENG vs. initial EMG: kappa value 0.164; ENG vs. follow-up EMG: kappa value 0.147). The prognostic quality of ENG and EMG was not influenced by the etiology of the palsy (Pearson's $\chi^{2}$ test, ENG $P=.432$; initial EMG $P=$.785; follow-up EMG $P=.772$ ).

\section{DISCUSSION}

Although electrical tests were already introduced in the 1970s, and many controversial studies concerning the prognostic value of each of these tests have followed, 2,4,10,11 we compared for the first time, to our knowledge, the usefulness of ENG and EMG to predict recovery versus defective healing directly in a large sample of patients with acute peripheral facial palsy. The retrospective design of the study is a limitation. A considerable source of bias because of missing data resulting from the exclusion of patients cannot be ruled out. The strong (strict/harsh) inclusion criteria ensured homogeneity of the data because the clinical outcome of all patients was known, yet this represents another source of bias because patients with severe lesions are more likely to reappear for control examinations. The considerably high rate of 67 (33\%) patients with some kind of defective healing at final presentation might thus be explained.

In most patients with acute facial palsy (idiopathic or Bell's palsy), an underlying cause cannot be found. In these patients, the chance of recovery is very high: complete recovery rates between $70 \%$ and $90 \%$ have been reported.9,12 Therefore, we do not need a prognostic tool to predict full recovery. A useful prognostic tool would help to identify the minority of patients who will develop defective healing. Chow et al. ${ }^{11}$ analyzed ENG results in Chinese patients with Bell's palsy. They estimated a sensitivity and specificity of using ENG to predict recovery of $82 \%$ and $100 \%$, respectively. These results are misleading, and the interpretation that ENG was a useful indicator has to be interpreted carefully. Using our methodologic approach focusing on defective healing, we calculated their PPV to predict defective healing that is only $65 \%$. Therefore, as was seen in our study, in a recent Korean study, ${ }^{4}$ ENG was not useful to predict defective healing in patients with Bell's palsy. Because Wallerian degeneration in the peripheral facial nerve takes approximately 72 hours to become apparent after onset of the lesion, it is recommended that ENG not be performed until at least 3 days after onset. In approximately $90 \%$ of our patients, we respected this rule. The cutoff point of $75 \%$ or less versus more than $75 \%$ amplitude loss to predict recovery versus defective healing is commonly accepted. ${ }^{4,10}$ Thus, we rule out methodologic flaws that might have weakened the ENG results. Unfortunately, we did not perform follow-up ENG examinations. Therefore, we cannot exclude the possibility that serial ENG examinations might improve its prognostic value.

In contrast, we confirmed the results of recent studies that showed that voluntary needle EMG, or, even better, serial EMG examinations, are a very useful tool to predict defective healing in acute facial palsy. ${ }^{1,5}$ For the 
initial EMG, we found a PPV and NPV of $91 \%$ and $89 \%$, respectively. It takes approximately 14 days until signs of degenerative lesions are detectable in the facial muscles. This is why the first EMG was not classifiable in some patients. We must be aware that a single EMG examination is not sufficient for some patients. Especially in traumatic lesions, the initial phase is critical. However, decision-making concerning decompression surgery solely by electrophysiologic tests is not advisable anyway. Our subset of patients with traumatic lesions was too small to allow further subanalysis. We recommend decompression surgery in accordance with the results of high-resolution computed tomography, indicating the fracture lines, and a history of early-onset palsy if ENG shows an amplitude loss more of than $75 \%$ and if voluntary EMG detects only single-fiber or no activity in all six important mimic muscles.

After a period of 15 days, PPV and NPV of follow-up EMG increased to $100 \%$ and $96 \%$. No other single tool with such high prognostic value has become available to date. It should be mentioned that needle EMG is a sensitive tool to detect regional signs of defective healing in the face, especially synkinetic activity. Therefore, EMG could be directly helpful in treatment planning, for instance, in giving Botulinum toxin treatment to suppress synkinetic movements.

\section{CONCLUSION}

In our series of 201 patients with acute peripheral facial palsies of different origins, needle EMG was more accurate for predicting the course of the palsy than ENG, especially when performed serially. The concordance between the EMG and ENG results were low. In analyzing the literature, EMG was shown to have better prognostic values than any other diagnostic tool. However, initial EMG results were not classifiable in one sixth of the patients. In these patients, ENG might be of additive value. We recommend that any physician treating patients with facial palsy be familiar with EMG and ENG and their advantages and disadvantages.

\section{BIBLIOGRAPHY}

1. Sittel C, Stennert E. Prognostic value of electromyography in acute peripheral facial nerve palsy. Otol Neurotol 2001;22: $100-104$

2. Esslen E. The acute facial palsies: investigations on the localization and pathogenesis of meato-labyrinthine facial palsies. Schriftenr Neurol 1977;18:1-164.

3. Sittel C, Guntinas-Lichius O, Streppel M, Stennert E. Variability of repeated facial nerve electroneurography in healthy subjects. Laryngoscope 1998;108:1177-1180.

4. Lee DH, Chae SY, Park YS, Yeo SW. Prognostic value of electroneurography in Bell's palsy and Ramsay-Hunt's syndrome. Clin Otolaryngol 2006;31:144-148.

5. Grosheva M, Guntinas-Lichius O. Significance of electromyography to predict and evaluate facial function outcome after acute peripheral facial palsy. Eur Arch Otorhinolaryngol 2007; Epub ahead of print.

6. House JW. Facial nerve grading systems. Laryngoscope 1983 93:1056-1069.

7. Guntinas-Lichius O, Streppel M, Stennert E. Postoperative functional evaluation of different reanimation techniques for facial nerve repair. Am J Surg 2006;191:61-67.

8. Seddon H. Three types of nerve injury. Brain 1943;66: 237-288.

9. Sittel C, Sittel A, Guntinas-Lichius O, Eckel HE, Stennert E. Bell's palsy: a 10-year experience with antiphlogisticrheologic infusion therapy. Am J Otol 2000;21:425-432.

10. May M, Blumenthal F, Klein SR. Acute Bell's palsy: prognostic value of evoked electromyography, maximal stimulation, and other electrical tests. Am J Otol 1983;5:1-7.

11. Chow LC, Tam RC, Li MF. Use of electroneurography as a prognostic indicator of Bell's palsy in Chinese patients. Otol Neurotol 2002;23:598-601.

12. Peitersen E. Bell's palsy: the spontaneous course of 2,500 peripheral facial nerve palsies of different etiologies. Acta Otolaryngol Suppl 2002:4-30. 